\title{
EDITORIAL SPECIAL ISSUE ON SERVICE SYSTEMS AND SERVICE MANAGEMENT
}

\author{
Chunguang Bai ${ }^{1}$ Junjie $\mathrm{Wu}^{2}$ Yongbo Xiao ${ }^{3}$ \\ ${ }^{I}$ School of Management and Economics, University of Electronic Science and Technology of China, Chengdu \\ 610054, China \\ chunguang.bai@gmail.com $(\bowtie)$ \\ ${ }^{2}$ School of Economics and Management, Beihang University, Beijing 100191, China \\ wujj@buaa.edu.cn \\ ${ }^{3}$ School of Economics and Management, Tsinghua University, Beijing 100084, China \\ xiaoyb@sem.tsinghua.edu.cn
}

Service system or service management is a value coproduction configuration of person, facilities, tools, organizational network, technology, internal and external service systems connected via value propositions, and shared information that satisfy the needs or aspirations of customers. The mankind has entered the era of globalization in 21 st century, the interdependence of things in service system becomes closer and the interaction is more complex. The service system is a complex system that is affected by the demand forecasting, customer relationship, public relations, customer behavior, satisfaction, and retention, service cost, profitability of service investment, risk, and so on. Some concepts, such as non-linearity, uncertainty, complexity, ambiguity, have appeared in service management. Some new theories and principles of service sciences appear, and the information technology and network technology develops rapidly. Different industries have encountered different problems of service management. Service management has a very complex process. Moreover, all processes must be coordinated across numerous service parts and multiple levels in the supply chain. As a managerial direction, service management has formed its system and has been study in various topics.

This special issue aims to invite famous researchers, practitioners and developers at home and abroad in service system or service management and communicate the new developments in service system or service management and its applications in society, economy and management. The topics of this issue include but are not limited to Theory and Principle of Service Sciences; Service System Design, Operations, and Management; Supply Chain Management for Service; Service Marketing and Financial Management; Specific Industrial Service Management; Service Information Technology and Decision Making; Service Empirical Studies and Case Studies; Management of healthcare services; Electronic business new models and strategies; Data analytics and service management. This special issue will be a good opportunity of international communication for experts in service system or service management. 
This special issue contains the selected extended papers presented at The 14th International Conference on Service Systems and Service Management (ICSSSM 2017) at Dalian, China in June 2017 as well as some new submissions. The 14th ICSSSM is jointly sponsored by IEEE Systems, Man and Cybernetics Society, The Chinese University of Hong Kong, Tsinghua University, and Dongbei University of Finance and Economics. It is hosted by the School of Management Science and Engineering, Dongbei University of Finance and Economics, Dalian. The main purpose of ICSSSM is to provide a platform for researchers, scholars, practitioners and students involved in the broad area of service systems and service management to disseminate their latest research results, as well as to exchange views on the future research directions. After the peer-review process, seven papers were accepted.

Niu, Liu, Chen and Ji (2018) examine the fashion brand's profit performances when the contract manufacturer is either an OEM (having no design capability) or an ODM (having design capability), and investigate the impact of the fashion brand's bargaining power on the buy-back price. The fashion brand is better off in the ODM scenario only when its bargaining power is sufficiently larger. The order quantity in the ODM scenario is always larger than that in the OEM scenario, and it is closer to the system optimal order quantity. Both the wholesale price and the buy-back price are decreasing in the fashion brand's bargaining power. From the perspective of environmental sustainability, the ODM scenario is worse than the OEM scenario.

Duan, Su, Zhu and Lu (2018) study on how to distribute the blood items among different departments within a hospital and investigates the allocation approach with the novel management method by centralizing the inventory of several different departments. By integrating the blood inventory requirements of some departments, the hospital could reduce the rate of blood shortage and wastage effectively, release the pressure of the occupancy of resources and reduce the bullwhip effect of blood products. This paper illustrates the centralization principle in hospital and formulates the mixed integer programming model to work out the optimal allocation network scheme and the optimal inventory setting for every department. And the results of the numerical example demonstrate that this centralization method could considerably reduce blood shortage and wastage in hospital by about $72 \%$ and $90 \%$ respectively.

Cai, Li, Dai and Zhou (2018) study the effect of online reviews on consumers' purchasing decisions as well as pricing strategies of manufacturers and the e-retailer. They use neo-Hotelling model to characterize consumer choice and induce demand functions of multiple products, and further employ a game theoretical approach to obtain equilibrium pricing strategies of manufacturers and the retailer. Information on quality and fit dimension provided by online reviews plays different role in affecting demands of products. Compared to the case without online reviews, online reviews improve a manufacturer's optimal wholesale price, profit as well as the retailer's optimal retailing price, when the adjusted quality revealed by reviews is high; however, online reviews can improve the e-tailer's optimal profit, only when the standard 
deviation of products' quality revealed by reviews is large enough. Last, the increase of competing manufacturers' quantity would change the dominance between two kinds of effect from reviews on supply chain members.

Wang, Qian, Li and Zhang (2018) provide a thorough experimental comparison of several well-known detectors, including supervised C4.5 and NB, unsupervised PCA and MDS, semi-supervised HySAD methods, as well as statistical analysis methods. MovieLens $100 \mathrm{~K}$ is the most widely-used dataset in the realm of shilling attack detection, and thus it is selected as the benchmark dataset. Meanwhile, seven types of shilling attacks generated by average-filling and random-filling model are compared in our experiments. Clearly causes and essential characteristics insider attackers might determine the success or failure of different kinds of detectors.

Liu, Wang, Wang, Zuo, Zhang and Zheng (2018) propose a novel topic model named hierarchical sparse NMF with orthogonal constraint (HSOC), which is based on non-negative matrix factorization and builds topic hierarchy via splitting super-topics into sub-topics. Extensive experimental results on real-world corpora show that the purposed model achieves comparable performance on topic quality and better performance on semantic feature representation of documents compared with baseline methods.

Yang, Wang and Chai (2018) develops manufacturer's channel selection models considering carbon emission reduction and remanufacturing. A higher recovery rate of remanufacturing can bring a higher reduction in carbon emission level and a lower price for consumers in the three channel structures. Compared to the pure online channel and pure offline channel, the dual channel is the optimal selection for a for-profit manufacturer. However, for a retailer, the pure offline channel is more profitable than the dual channel.

Wang and Tang (2018) developed a multi-objective optimization model for a seru production system formation problem to minimize the cost and maximize the service level of the system. The purpose of this paper is to formulate a robust production system that can respond efficiently to the stochastic demand. Sample average approximation (SAA) is used to approximate the expected objective of the stochastic programming. The non-dominated sorting genetic algorithm II (NSGA-II) is improved to solve the multi-objective optimization model.

\section{References}

[1] Cai, X.Y., Li, J.B., Dai, B. \& Zhou, T. (2018). Pricing strategies in a supply chain with multi-manufacturer and a common retailer under online reviews. Journal of Systems Science and Systems En gineering, 27 (4): 435-457.

[2] Duan, J.N., Su, Q., Zhu, Y.H. \& Lu, Y.S. (2018). Study on the centralization strategy of the blood allocation among different departments within a hospital. Journal of Systems Science and Systems En gineering, 27 (4):417-434.

[3] Liu, R., Wang, X.G., Wang, D.Q., Zuo, Y., Zhang, H. \& Zheng, X.Z. (2018). Topic splitting: a hierarchical topic model based on non-negative matrix factorization. 
Journal of Systems Science and Systems En gineering, 27 (4): 479-496.

[4] Niu, B.Z., Liu, Y.Q., Chen, L. \& Ji, P. (2018). Outsource to an OEM or an ODM? Profitability and sustainability analysis of a fashion supply chain. Journal of Systems Science and Systems Engineering, 27 (4): 399-416.

[5] Wang, Y. \& Tang, J.F. (2018). Manufacturer's channel selection considering carbon emission reduction and remanufacturing. Journal of Systems Science and Systems Engineering, 27 (4): 519-537.

[6] Wang, Y.Q., Qian, L.Q., Li, F.Z. \& Zhang, L. (2018). A comparative study on shilling detection methods for trustworthy recommendations. Journal of Systems Science and Systems Engineering, 27 (4): 458-478.

[7] Yang, L., Wang, G.Y. \& Chai, Y.N. (2018). Manufacturer's channel selection considering carbon emission reduction and remanufacturing. Journal of Systems Science and Systems Engineering, 27 (4): 497-518

Chunguang Bai is currently a professor in The School of Management and Economics at University of Electronic Science and Technology of China. She earned her Ph.D. in management science from the Dalian University of Technology at China in 2010. Her research interests include sustainable supply chain management, management of technology, and business process management and the environment. She has published over 30 papers on English journals such as European
Journal of Operational Research and International Journal of Production Economics, International Journal of Production Research, Supply Chain Management: An International Journal, and the Journal of Cleaner Production, Annals of Operations Research, etc. She has over 1800 citations in Google Scholar. Two of her papers has become some of highest cited in ten years (ESI index). She was nominated as Chinese Most Cited Researchers by Elsevier (2014-2017). Her research has been supported by the National Natural Science Foundation of China Project (71102090,71472031,71772032); the Liaoning Excellent Talents in University (WJQ2014029), etc.

Junjie Wu is currently a full professor in Information Systems Department, School of Economics and Management, Beihang University. He is also the director of the Research Center for Data Intelligence (DIG), the vice director of Beijing Key Laboratory of Emergency Support Simulation Technologies for City Operations, and a senior researcher in Beijing Innovation Center for Big Data and Brain Computing. His general area of research is data mining and machine learning, with a special interest in social, urban and financial computing. $\mathrm{He}$ is the PI of one NSFC key project (IoT Big Data Mining), one MOST 863 project (Social Computing), and various MIIT projects (Security Computing). He has published one monograph in Springer and over 100 papers in refereed conference proceedings (e.g., KDD, IJCAI, AAAI) and prestigious journals (e.g., ISR, TKDE, TIP). $\mathrm{He}$ is the recipient of various nation-wide academic awards in China, including the NSFC Distinguished 
Young Scholars, the MOE Changjiang Young Scholars, and the National Excellent Doctoral Dissertation.

Yongbo Xiao is an associate professor (with tenure) at School of Economics and Management, Tsinghua University, China. He received his Ph.D. and M.A. in management science and engineering in 2006, and B.E. in Management Information Systems in 2000, all from Tsinghua University. He joined Tsinghua SEM as an assistant professor in Aug. 2008 after he completed his postdoctoral research in Department of Economics in Tsinghua SEM. He was awarded the "Science Fund for Excellent Young Scholar" under National Natural Science Foundation of China (NSFC) in 2012 and the "Young Scholar Award of Chinese Management Science" in 2014. He was elected as a Chang Jiang scholar in 2016. Dr. Xiao's research interests include revenue and pricing management, service management, supply chain management, and healthcare management. 